\title{
Bicycling about to be explained?
}

A new calculation seems to have made the behaviour of bicyclists explicable — but there is a long way to go before the matter will be satisfactorily understood.

THE essential problem of the bicycle - why does a moving bicycle not tip over more often? - may at last have been solved.

That at least is the implication of a report by G. Franke, W. Suhr and F. Riess from the University of Oldenburg (Eur. J. Phys. 11, 116; 1990). But one should be cautious. In the long history of mechanics, claims that the problem of the bicycle has been solved have been regularly followed by demonstrations that the claims are based on over-simple approximations that invalidate the conclusions. Ominously, even Franke and his colleagues have simplified the problem by supposing that the rider steers the bicycle only by adjustments of the position of his or her centre of gravity. It is hands-off riding. On the face of things, the solution does not accommodate what common observation demonstrates - that riders pedalling find it advantageous to make energetic movements from side to side while playing with the direction of the front wheel.

Franke and his colleagues are forgiving about previous attempts at the problem, which they acknowledge to be difficult.

They also take note of earlier demonstrations that one of the crucial determinants of stability is the distance between the axis of the steering column and the centre of the front (steerable) wheel, called the 'trail length' in the argot of the discipline. This is why the front fork of a bicycle, the device that supports the steering mechanism and the front of the bicycle frame on the front wheel, is usually bent forward towards the bottom. Their objective is to solve the problem of the moving bicycle with as few limitations as they can manage. Among other things, for example, they have allowed for wheels of unequal radius, which means that their solution should be as easily applicable to penny-farthings as to modern designs.

Much of the interest in the new attempt at the problem is the manner in which the authors have formulated it. At least superficially, it is not as complicated as might be expected.

Crudely, if a bicycle (with its rider) were a rigid body, it would have six degrees of freedom (three translation degrees of freedom and three rotations). But bicycles are not rigid: the front wheel can be steered (through a 'steering angle') while the two wheels can rotate. So there are nine degrees of freedom altogether - six intrinsic to a rigid body and three strictly internal degrees of freedom. The con- figuration of the bicycle at any time could in principle be defined by specifying nine variables. If the bicycle is moving, for each degree of freedom there will be a coordinate in a suitably chosen reference frame and a corresponding velocity.

But it is clear that not all possible motions correspond to stable reality. To achieve that, the two wheels, for example, must remain in contact with the ground. So the problem of the moving bicycle is that of the motion of a rigid body which is subject to what are called constraints. The simplest way of dealing with such problems is to choose a set of coordinates that will automatically ensure that the constraints are satisfied, which sometimes requires a little ingenuity.

But Franke and his colleagues make the task seem simple. Of the nine configurational degrees of freedom, for example, two are eliminated by the requirement that the wheels should be on the ground, while - at least on level ground - neither the absolute degree of rotation of the two wheels nor the absolute position and direction of the frame can be relevant to stability.

The result is that the configuration of a bicycle can be described by only two variables, taken as the steering angle and the lean angle. Of course, the dimensions of the actual bicycle enter as parameters Franke and his colleagues use the radii of the two wheels, the trail length, the distance along the perpendicular between the centre of the rear wheel and the steering axis and the distance between the centre of the front wheel and that same perpendicular as the parameters that specify the bicycle. By similar arguments, the dynamical degrees of freedom are reduced to three - the time derivates of the steer angle and the lean angle and the scalar velocity of the bicycle (measured, for the sake of definiteness, at the rear wheel).

Turning this description of a moving bicycle into a set of equations of motion that might be solved is the more tricky part of the calculation. For practical purposes, the authors used a moving system of rectangular coordinates whose origin is the point at which the steering axis meets the ground, but whose unit vectors are along the direction of motion of the rear wheel, along the point of contact at the rear wheel with the ground to its centre and towards the hub of the rear wheel. (The last choice leads to the convenient result that the lean angle does not appear in the equations of motion.) Even so, the outcome is a vector equation (equivalent to three simultaneous scalar equations) that cannot be solved analytically, only numerically. No doubt these equations will soon be known by heart in the places at which bicycles are designed; they are a potentially endless resource for bicycle-makers.

The immediate benefits of the investigation, unfortunately, are more a spur to further investigation than a revelation in themselves. Franke and his colleagues have allowed for the bicycle rider to adjust his position laterally relative to frame. The rider's weight is supposed to be an unladylike $70 \mathrm{~kg}$. One set of solutions to the equations of motions arises by supposing that a no-hands bicyclist of this size travels in a repeating circle, with a constant steering angle and a constant velocity.

Indeed, as one would expect, the greater the lean angle, the greater must be the steering angle (for a fixed velocity) or the greater the velocity (for a fixed steering angle). It is more surprising that while, above a certain critical velocity, there should be only one combination of steering angle and rider displacement that will keep the bicycle travelling in a circle at a prescribed lean angle, below that critical speed, there are two lean angles at which the same rider displacement will keep the bicycle in a circle. Those who ask whether this is a sign of incipient bifurcation to chaos will not be surprised to be answered in the affirmative.

Perhaps the most practical implication of the calculations so far carried out is that the speed at which circular motion can be stable is sensitively dependent on the moment of inertia of the wheels: the smaller the combined moment of inertia, the greater must be the velocity. Similarly, the trailing distance crucially determines the range of velocities over which motion in a circle can be stable; the greater the forward displacement of the front wheel, the greater the range of stable velocity. That, of course, is what all circus bicycle-riders know by instinct.

What all this means for real bicycleriders is hard to tell. That the forward displacement of the rider's centre of gravity is the equivalent of lengthening the trailing distance is unsurprising.

This, no doubt, is why riders stand on the pedals when going very slowly. But what the new equations of motion mean for the behaviour of bicycle-riders remains to be determined. John Maddox 